\title{
Estudo etnobotânico do conhecimento e uso das plantas medicinais no município de Buriticupu, Maranhão, Brasil
}

O uso das plantas para fins terapêuticos é uma prática tradicional muito difundida em diversas populações, com especificidades de cada região. Tal prática está presente desde os primórdios das civilizações, sendo o seu conhecimento repassado durante as gerações. Esta pesquisa teve como objetivo fazer um levantamento sobre plantas medicinais utilizadas pelos moradores da área urbana do município de Buriticupu/MA. Foram entrevistados 110 pessoas por meio de questionários semiestruturados. Foram levantadas 82 espécies medicinais distribuídas em 43 famílias botânicas. As famílias Lamiaceae e Fabaceae foram as mais citadas e as espécies Hortelã (M. crispa), Capim-Santo (C. citratus), Erva-Cidreira, (H. atrorubens), Malva-do-Reino (M. sylvestris) e Folha Santa (K. pinnata) apresentaram maior número de citações, sendo que M. arvensis (vique) foi a única que obteve FRIPS com $100 \%$. As folhas (63\%) foram a parte botânica mais usada nos preparos caseiros realizados predominantemente por decocção. Dentre as principais afecções tratadas por meio das plantas medicinais, as mais prevalentes foram infecciosas e parasitárias (30\%), as doenças do sistema digestivo (15\%) e sistema reprodutor feminino (15\%). Os resultados das correlações entre o número de filhos e a idade estão positivamente ligados ao número de plantas citadas pelos informantes, enquanto que o número de plantas citadas é influenciado negativamente pela idade $(p<0,05)$. Este estudo confirma que os moradores da região urbana do município utilizam com frequência plantas medicinais como forma de tratamento para suas doenças mais comuns.

Palavras-chave: Conhecimento Tradicional; Medicina Popular; Etnobiologia.

\section{Ethnobotanical study of knowledge and use of medicinal plants in Buriticupu, Maranhão, Brazil}

\begin{abstract}
The use of plants for therapeutic purposes is a traditional practice widespread in different populations, with specificities of each region. This practice has been present since the dawn of civilizations, and its knowledge has been passed on through the generations. This research aimed to make a survey on medicinal plants used by residents of the urban area of Buriticupu/MA. 110 people were interviewed through semi-structured questionnaires. A total of 82 medicinal plant species were recorded distributed in 43 botanical families. The Lamiaceae and Fabaceae families were the most cited and the species Hortelã (M. crispa), Capim Santo (C. citratus), Erva-cidreira (H. atrorubens), Malva-do-reino (M. sylvestris) and Folha-santa (K. pinnata) presented the highest number of citations, and M. arvensis (vique) was the only one to obtain $100 \%$ FRIPS. The leaves (63\%) were the most used botanical part in homemade preparations made predominantly by decoction. Among the main diseases treated by medicinal plants, the most prevalent were infectious and parasitic (30\%), digestive system diseases (15\%) and female reproductive system (15\%). The results of correlations between number of children and age are positively linked to the number of plants cited by the informants, while the number of plants cited is negatively influenced by age $(p<0.05)$. This study confirms that urban residents often use medicinal plants as a treatment for their most common diseases.
\end{abstract}

Keywords: Traditional Knowledge; Folk Medicine; Ethnobiology.

Topic: Uso de Recursos Naturais

Reviewed anonymously in the process of blind peer
Received: 02/10/2019

Approved: 26/11/2019
Elisama de Melo Alencar

Instituto Federal do Maranhão, Brasil

http://lattes.cnpq.br/4982866739188241

elisamamelodealencar@gmail.com

Reinaldo Lucas Cajaiba (iD

Instituto Federal do Maranhão, Brasil

http://lattes.cnpq.br/4059139430516677

http://orcid.org/0000-0003-0176-9201

reinaldocajaiba@hotmail.com

Jesuino da Silva Costa Martins (it

Instituto Federal do Maranhão, Brasil

http://lattes.cnpq.br/0872704726864852

http://orcid.org/0000-0003-2137-0054

elisamamelodealencar@gmail.com

\section{Rogério Soares Cordeiro \\ Instituto Federal do Maranhão, Brasil \\ http://lattes.cnpq.br/1588494367215663 \\ rogerio.cordeiro@ifma.edu.br \\ Elson Silva de Sousa (iD \\ Instituto Federal do Maranhão, Brasil \\ http://lattes.cnpq.br/0079132488060600 \\ http://orcid.org/0000-0002-7369-3704 \\ elson.silva@ifma.edu.br}

Vilson de Almeida Sousa

Instituto Federal do Maranhão, Brasi

http://lattes.cnpq.br/1956408131769136

vilson.almeida@ifma.edu.br

\section{Referencing this:}

ALENCAR, E. M.; CAJAIBA, R. L.; MARTINS, J. S. C.; CORDEIRO, R. S ; SOUSA, E. S.; SOUSA, V. A.. Estudo etnobotânico do conhecimento e uso das plantas medicinais no município de Buriticupu, Maranhão, Brasil. Revista Ibero Americana de Ciências Ambientais, v.10, n.6, p.328-338, 2019. DOI: http://doi.org/10.6008/CBPC21796858.2019 .006 .0028 


\section{INTRODUÇÃO}

Desde a sua existência, o homem sempre buscou nos recursos naturais uma alternativa para a cura e/ou alívio de suas doenças, com o objetivo de melhorar sua qualidade de vida e aumentar suas chances de sobrevivência (CARVALHO et al., 2010; GIRALDI et al., 2010). As plantas medicinais para tais fins, tornou-se uma prática muito difundida entre comunidades de todo mundo e perpassa para os dias atuais (COSTA, 2013).

O conhecimento empírico sobre as plantas medicinais no decorrer da história da humanidade, se mostra eficaz no que se refere aos cuidados imediatos à saúde (FLOR et al., 2015). Segundo a Organização Mundial de Saúde (OMS), 80\% da população mundial dependem das práticas tradicionais com relação a atenção primária à saúde, e desses, $85 \%$ faz uso de alguma espécie de planta ou preparações tendo como base vegetais (BRASIL, 2006). Desse modo, a busca por alternativas terapêuticas, como as plantas medicinais, torna-se muitas vezes o principal e único recurso natural disponível e acessível para algumas comunidades e grupos étnicos (BARBOSA et al., 2010; WANDERLEY et al., 2015).

Mesmo com os avanços da medicina moderna, o uso de plantas medicinais por populações indígenas, quilombolas e comunidades rurais ainda é uma alternativa importante no tratamento de doenças, e os conhecimentos tradicionais adquiridos a partir dessa prática é transmitido ao longo dos anos através das gerações, geralmente de forma oral (NETO et al., 2014). Segundo Oliveira (2016), é através da observação de práticas como essas que surge um conhecimento rico em fusões culturais, religiosas e étnicas que a muito tempo é utilizado para o tratamento de doenças.

Dessa forma, o registro do conhecimento tradicional torna-se imprescindível, uma vez que informações sobre o uso empírico das plantas se encontram ameaçados de desaparecimento (CAJAIBA et al., 2016a). Portanto, o resgate e a valorização desses conhecimentos necessitam ser preservados. Assim, essas comunidades se tornam o principal objeto de estudo Etnobotânico (NETO et al., 2014).

A Etnobotânica é uma das áreas da ciência que estuda a interação entre o homem e as plantas, podendo ser definida, de acordo com Rocha et al. (2015), como "o estudo da relação existente entre o Homem e as Plantas e o modo como essas plantas são usadas como recursos". Dessa forma, permite entender as maneiras pelas quais as pessoas classificam, manipulam e utilizam certas espécies de plantas (ROCHA et al., 2015).

No Maranhão, estudos Etnobotânicos são escassos, sendo assim, objetivou-se com esta pesquisa conhecer as principais espécies de plantas medicinais utilizadas pelos moradores da área urbana do município de Buriticupu, estado do Maranhão, além de saber as principais técnicas, formas de uso, partes mais utilizadas e quais principais doenças são tratadas através do uso, assim como, a forma de obtenção de conhecimento do uso dessas espécies. 


\section{MATERIAIS E MÉTODOS}

\section{Local de estudo}

O estudo foi desenvolvido na área urbana do município de Buriticupu, situado na região da Amazônia Maranhense, a aproximadamente $430 \mathrm{~km}$ da capital São Luís. O mesmo possui extensão territorial de 2.545,44 km² e uma população estimada em de 71.723 habitantes (IBGE, 2018). O clima da região é quente e úmido, uma subdivisão do clima tropical e apresenta duas estações bem definidas, um período chuvoso, de dezembro a junho e o período de estiagem, de julho a novembro. A temperatura média anual varia de 25 a 27 으 e a umidade relativa do ar é de $80 \%$, em média, enquanto os índices pluviométricos estão entre 1.800 e $2.000 \mathrm{~mm}$ (CAJAIBA et al., 2019).

O município encontra-se inserido no ambiente geológico conhecido como Bacia Sedimentar do Pindaré, que atinge 12,40\% das Bacias do Estado do Maranhão. O relevo é constituído por formações de tabuleiros, separados em faixas por drenos e grotões, situado a uma altitude de $200 \mathrm{~m}$ acima do nível do mar (LIMA et al., 2017).

\section{Coleta dos dados}

A coleta das informações consistiu na aplicação de entrevistas semiestruturadas, tendo como base o método de geração de dados (POSEY, 1987), a moradores da área urbana do município de Buriticupu, entre os meses de junho e setembro de 2019. Participaram da pesquisa pessoas de ambos os sexos com idade igual ou superior a 18 anos. Para as respostas dos entrevistados se considerou a máxima fidelidade possível no momento da escrita, sendo, posteriormente, organizadas em um banco de dados padronizado no Microsoft Office Excel $^{\circ} 2016$.

Foram coletadas somente espécies citadas pelos informantes e quando disponíveis. Os exemplares foram fotografados e coletados pelos pesquisadores, sempre que possível com suas flores e/ou frutos necessário para a identificação taxonômica. Os exemplares foram depositados no Laboratório de Biologia do Instituto Federal do Maranhão e uma parte deles foram comparados com exsicatas da Universidade Federal do Pará. A identificação taxonômica foi realizada com auxílio de bibliografia especializada: Lorenzi et al. (2008), pelas bases: International Plant Name Index (IPNI) ${ }^{1}$, Tropicos ${ }^{2}$, Plant Taxonomy Database ${ }^{3}$ e site da Flora Brasileira ${ }^{4}$.

Os nomes das espécies e famílias botânicas foram atualizados de acordo com a base de dados da Lista de Espécies da Flora do Brasil ${ }^{5}$ e do Missouri Botanical Garden Tropicos. Conforme instruções da Resolução 466/12 para pesquisas com seres humanos, todas as pessoas que concordaram a participar da pesquisa assinaram um Termo de Consentimento Livre e Esclarecido (TCLE). Foram amostrados cinco bairros da área urbana do município de Buriticupu. Os bairros selecionados representam os diferentes estratos

\footnotetext{
1 http://www.ipni.org

2 http://www.tropicos.org

3 http://www.ethnomedicinals.com/searcheng.html

${ }^{4}$ http://www.ibama.gov.br/flora/extinção.html

5 http://www.floradobrasil.jbrj.gov.br
} 
sociais do município em questão. Para definir o tamanho da amostra de cada bairro, considerou o tamanho populacional com base no método aplicado por Gil (2008), e calculado de acordo com a seguinte fórmula: $n=$

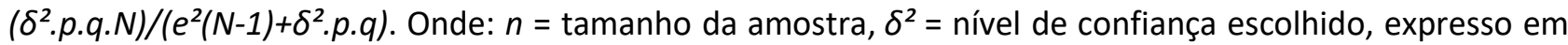
número de desvio-padrão, $p=$ porcentagem, com a qual o fenômeno se verifica, $q=$ porcentagem complementar, $N=$ tamanho da população, $e^{2}=$ erro máximo permitido. A margem de confiança adotada foi de $95 \%$.

Para selecionar as residências a serem entrevistadas, foi utilizado a amostragem sistemática proposta por Gil (2008), através da seguinte equação: $K=N / n$. Onde: $K=$ intervalo de seleção, $N=$ número de residências do bairro, $n=$ número de casas da amostra. A definição do ponto de partida foi por sorteio aleatório simples através do $K$ primeiro da relação. A partir daí, selecionou os indivíduos sistematicamente a cada intervalo de tamanho.

\section{Análise dos dados}

Foi calculado o número de vezes que os indivíduos de cada espécie foram citados (frequência absoluta) e a proporção do número de observações de uma determinada espécie em relação ao total de observações (frequência relativa), sendo expressa em termos percentuais (SOUSA et al., 2019). Calculou-se também, a Frequência de indicação do problema para a espécie (FRIPS), que é resultante da proporção entre a frequência de indicação do problema de saúde (FIPS), pela frequência de citação da espécie correspondente (FCE), através da seguinte fórmula: FRIPS = (FIPS/FCE) X 100. Isto corresponde ao que Amorozo et al. (1988) denominaram "concordância de uso popular" (CUP). Quanto maior a FRIPS, maior a concordância de uso entre os informantes.

Foi aplicada uma correlação de Spearman para verificar a relação entre o número de plantas citadas com escolaridade, idade e número de filhos. A diferença entre plantas citadas por homens e mulheres foi comparada através do teste não paramétrico do Qui-quadrado, analisado no programa estatístico Past 3.1 (HAMMER et al., 2001). As doenças/sintomas citadas pelos entrevistados foram categorizadas de acordo com a Classificação Estatística Internacional de Doenças e Problemas Relacionados à Saúde (CID-10 2013, do inglês International Statistical Classification of Diseases and Related Health Problems - ICD).

\section{RESULTADOS}

Foram entrevistadas 110 pessoas, destas, $79(88 \%)$ pertenciam ao sexo feminino. A idade dos informantes variou entre 19 a 86 anos, com média de 52,05 (dp $\pm 16,07)$. Quanto à escolaridade, 47\% eram analfabetos e apenas $15 \%$ tinham o ensino médio completo. Verificou-se que a maioria dos entrevistados (87\%) declararam ser oriundos do próprio estado do Maranhão, sendo que $42 \%$ residem no município há mais de 20 anos. A profissão mais predominante entre a maioria dos moradores entrevistados foi a de agricultor (49\%). Observou-se que 59 das famílias entrevistadas (54\%) tinham menos de cinco filhos.

Sobre a obtenção das plantas para consumo, $57 \%$ dos entrevistados responderam que obtêm no quintal de suas residências, $15 \%$ compram e $11 \%$ obtêm com familiares. Outro aspecto observado foi a 
quantidade de vezes por ano que essas famílias utilizam as plantas medicinais, em suma, respostas como 'sempre' foram as mais comuns (47\%) e apenas $16 \%$ especificaram, em números, quantas vezes por ano utilizam tais plantas (Figura 1).

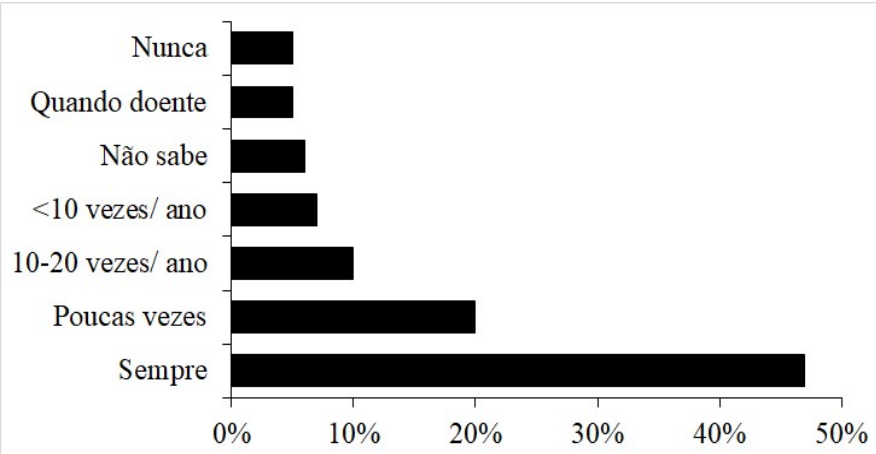

Figura 1: Quantidade de vezes por ano que os entrevistados e/ou membros das famílias utilizam plantas medicinais.

Entre os principais fatores que levam os entrevistados a usarem as plantas medicinais no tratamento de suas doenças ou de familiares, estão: costume $(20 \%)$, baixo custo $(17 \%)$ e por ser natural $(17 \%)$. Outros fatores determinantes para a escolha foram a ausência de efeitos colaterais (14\%) e a facilidade de acesso (14\%). Houve aqueles que disseram utilizar as plantas medicinais por algum tipo de crença (13\%), acreditando que estas possam ter algum poder divino. Apenas $1 \%$ declarou não acreditar nos benefícios da utilização de certas espécies de plantas para uso medicinal.

Em relação ao tipo de fonte para obtenção de conhecimentos sobre as plantas medicinais, 54\% responderam obter através de familiares. Outras fontes informadas pelos entrevistados foram: experiência própria (13\%), amigos (10\%), livros (7\%) e/ou internet (6\%). Quanto ao repasse dos conhecimentos tradicionais sobre as plantas medicinais, $85 \%$ afirmaram que repassam esses conhecimentos, destes, apenas $32 \%$ disseram passar para algum membro da família, enquanto $14 \%$ repassam para amigos ou conhecidos e $13 \%$ para seus vizinhos; $13 \%$ responderam que não costumam ou não repassariam tais conhecimentos para outras pessoas.

Tabela 1: Usos principais, nome popular/científico e Frequência relativa de indicação para cada espécie (FRIPS)

\begin{tabular}{|l|l|l|l|}
\hline Espécie & Nome científico & Indicação & FRIPS \\
\hline Vique & M. arvensis & Gripe & $100 \%$ \\
\hline Gengibre & Z. officinale & Infeção & $91 \%$ \\
\hline Malva-do-reino & M. sylvestris & Gripe & $86 \%$ \\
\hline Boldo & P. barbatus & Fígado & $81,22 \%$ \\
\hline Erva-cridreira & H. atrorubens & Calmante & $74,27 \%$ \\
\hline Hortelã & M. crispa & Gripe & $69,53 \%$ \\
\hline Folha-santa & K. pinnata & Anti-inflamatório & $68,80 \%$ \\
\hline Capim-santo & C. citratus & Calmante & $59,71 \%$ \\
\hline Mastruz & C. ambrosioides & Anti-inflamatório & $53 \%$ \\
\hline
\end{tabular}

As plantas medicinais registradas pelos entrevistados foram representadas por 82 espécie distribuídas em 43 famílias botânicas. As famílias com maior número de espécies foram: Lamiaceae ( 9 espécies), Fabaceae (6 espécies), Asparagaceae, Myrtaceae e Zingiberaceae com 4 espécies cada. Sendo que, as espécies mais citadas foram: Hortelã (Mentha crispa, 34 citações), Capim-Santo (Cymbopogon citratus, 31 citações), Erva-Cidreira (Hyptis atrorubens, 22 citações). As espécies Malva-do-Reino (Malva sylvestris) e 
Folha Santa (Kalanchoe pinnata) obtiveram 20 citações cada. A frequência relativa de indicação para cada espécie (FRIPS), apresentou valores acima de 50\% para nove espécies, mas apenas a espécie Mentha arvensis (vique) obteve FRIPS de 100\%, sendo indicada para o tratamento de gripe (TABELA 1).

A folha foi a parte vegetal mais citada pelos entrevistados (63\%) como utilizada nas preparações dos remédios caseiros. Outras partes citadas foram: caule (9\%), flor (8\%), casca (6\%), sementes (5\%), fruto (4\%), entrecasca (4\%), e raiz (1\%). Também foi citado o uso de rizoma, bulbo e bulbinho, no entanto, essas foram incluídos dentro da categoria caule.

Dos diversos modos de preparo das espécies para consumo, o chá foi o principal, aparecendo em $54 \%$ das citações, seja este por infusão e/ou decocção, seguido de garrafada (14\%), sumo (10\%), lambedor (8\%), in natura (7\%); molho, suco e óleo somam 7\%. A ingestão (85\%) foi a forma mais frequente de uso entre as espécies medicinais. Outros modos de uso foram: uso tópico (8\%), banho de imersão (5\%) e nebulização (1\%). As indicações terapêuticas tratadas a partir do uso das 82 espécies de plantas medicinais citadas pelos entrevistados foram agrupadas em 11 categorias de sintomas/doenças conforme o CID-10, sendo que as mais prevalentes foram as Doenças infecciosas parasitárias-DIP (30\%) e as do Sistema Digestório-SD e Sistema Reprodutor Feminino-SRF com 15\% das cada (Figura 2).

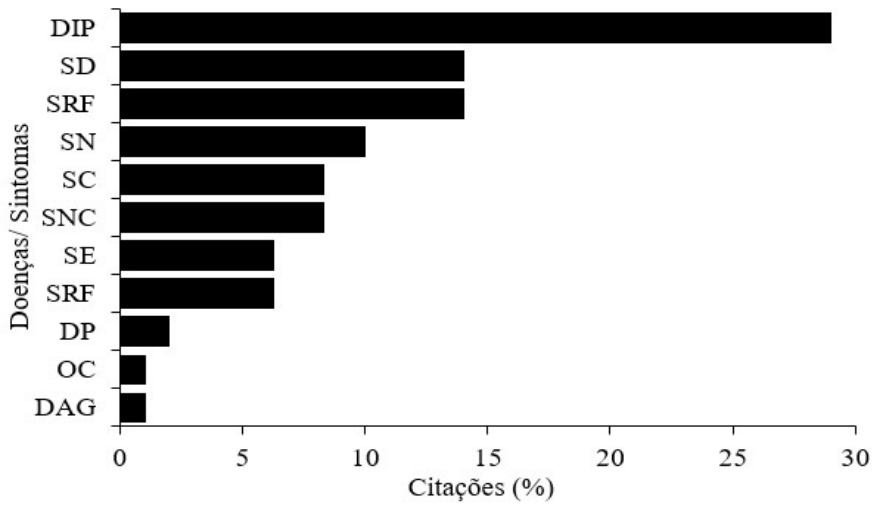

Figura 2: Principais afeç̧ões tratadas por plantas medicinais no município de Buriticupu/MA categorizadas segundo o CID 10. Doenças infecciosas e parasitárias (DIP); Sistema Digestório (SD); Sistema Reprodutor Feminino (SRF); Sistema Nervoso (SN); Sistema Cardiovascular (SC); Sintomas não classificados (SNC); Sistema Endócrino (SE); Doença de Pele (DP); Ossos e Cartilagens Articular (OC); Doenças do aparelho geniturinário (DAG).

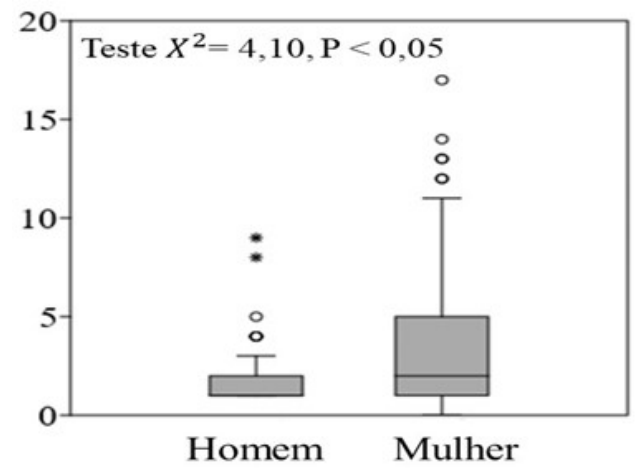

Figura 3: Nível de conhecimento e uso de plantas medicinais entre homens e mulheres participantes da entrevista.

A correlação de Spearman demonstrou que houve relação positiva entre o número de plantas citadas com números de filhos por residência $\left(R_{s}=0,632, p<0,01\right)$, idade $\left(R_{s}=0,395, p<0,05\right)$ e o tempo de estudo $\left(R_{s} 0,2385, p<0,05\right)$. Verificou-se através do teste do Qui-quadrado que houve diferença no nível de 
conhecimento entre homes e mulheres (Teste $\chi^{2}=4,10, \mathrm{P}<0,05$ ), sendo que as mulheres conhecem mais plantas com fins medicinais, entretanto, percebeu-se que mesmo em menor quantidade alguns homens citaram um número considerável de espécies, quando comparado às citadas pelas mulheres (Figura 3).

\section{DISCUSSÃO}

A utilização das plantas medicinais para fins terapêuticos é uma prática que vem sendo comprovada por diversos trabalhos realizados na área da Etnobotânica (VÁSQUEZ et al., 2014; CAVALCANTE et al., 2017; GONÇALVES et al., 2018). Os resultados obtidos nesta pesquisa, corroboram com tais, uma vez que foi verificado que a maioria dos entrevistados (98\%) afirmam fazer uso das plantas medicinas como uma alternativa primária no tratamento de doenças, devido principalmente, ao seu baixo custo e por serem consideradas de origem natural. Como o acesso aos serviços de saúde e aos fármacos não é possível para as populações mais carentes, a tendência é, que os mesmos recorram às plantas medicinais, por serem de fácil obtenção, além da tradição de serem benéficas e seguras (CAVALCANTE et al., 2017).

Entre as 82 espécies medicinais levantadas, as famílias botânicas Lamiaceae, Fabaceae, Asparagaceae, Myrtaceae e Zingiberaceae obtiveram maior representatividade. Lamiaceae foi a família botânica que apresentou maior quantidade de espécies citadas, em consonância com outros estudos realizados (FREITAS et al., 2012; ALVES et al., 2013; SANTOS et al., 2016). Esse fato pode ser justificado pela expressiva biodiversidade das espécies pertencentes a essa família, que são cosmopolitas e possuem uma grande importância terapêutica (PINTO et al., 2006; ALVES et al., 2013; FLOR et al., 2015). Lamiaceae e Fabaceae também fazem parte das famílias botânicas com maior número de espécies medicinais, além constituírem um grande do grupo de angiospermas (GUARIN NETO et al., 2003).

Hortelã (M. crispa), Capim-Santo (C. citratus.), Erva-Cidreira (H. atrorubens), Malva-do-Reino (M. sylvestris), Folha Santa (K. pinnata) e Vique (M. arvensis) foram as espécies mais citadas, corroborando com os resultados obtidos por Gonçalves et al. (2018) e Stefanello et al. (2018). Quanto a Frequência de Indicação apenas a espécie Vique (M. arvensis) apresentou FRIPS de 100\%, sendo indicado no tratamento de gripe. Esses dados ajudam na comprovação da eficácia de uma espécie medicinal, pois quanto mais informantes concordam com um determinado uso, maior será a validação desse conhecimento, podendo servir de base para estudos futuros, direcionados a farmacologia, melhorias dos medicamentos que já existem e até mesmo a cura de doenças (ROQUE et al., 2010; ZAGO, 2018).

Em concordância com pesquisas semelhantes (VIEIRA et al., 2015; BRITO, 2015; SANTOS et al., 2016), a parte vegetal mais utilizada no preparo dos remédios caseiros foram as folhas. O caule e as flores também obtiveram um grande número de citações, embora as flores, geralmente, sejam retiradas parcialmente. 0 uso predominante das folhas pode ser explicado devido a facilidade de coleta e por serem encontradas, praticamente, todo o ano, diferente das flores, frutos e sementes, disponíveis em época específica do ano (SILVA et al., 2017). A expressiva utilização das folhas representa uma prática de manejo sustentável da flora, reduzindo os impactos sobre as populações das espécies utilizadas (MESSIAS et al., 2015).

O chá foi a técnica mais utilizada para o preparo dos remédios caseiros, seja ela por decocção ou 
infusão. E a forma mais frequente de uso foi a ingestão. Observou-se que para os informantes, há preferência na preparação dos chás por decocção, independente da parte botânica utilizada. A facilidade nas preparações por decocção a torna uma das principais práticas empregada no preparo de medicamento no sistema de cura tradicional ${ }^{6}$ (SEGAWA, 2007; ROKAYA et al., 2010). A técnica consisti na fervura do material vegetal em água, até redução da mesma, a um quarto do volume original. A ingestão, enquanto forma de uso, obteve bastante citações, corroborando com estudos realizados por Lemos et al. (2015).

Os resultados demonstraram que as doenças mais tratadas a partir do uso das espécies medicinais pelos moradores foram as doenças parasitárias e infecciosas, doenças do sistema digestório e as doenças relacionadas ao sistema reprodutor feminino, sendo que, as doenças mais prevalentes foram as parasitárias e infecciosas, indicadas, também, nas pesquisa de Silva et al. (2017) e Gonçalves et al. (2018). Esses resultados podem relacionar-se a ausência de um sistema saneamento básico, uma vez que no município não há um sistema de tratamento de água, esgoto e coleta resíduos (PINTO et al., 2006; CAJAIBA et al., 2016b). Em geral, os sintomas atrelados a estas classes estão relacionados com os problemas primários de saúde, que são tratados primeiramente com o uso de plantas medicinais e sem auxílio do médico (POSSAMAI, 2000). Ressalta-se, que esses tipos de doenças são comuns, principalmente nas regiões do interior do país, assim como em outros países da América Latina (BENNETT et al., 2000).

Em relação ao gênero dos entrevistados, foi possível observar que as mulheres possuem um nível maior de conhecimento referente às espécies medicinais. Acredita-se que o conhecimento predominante das mulheres sobre as espécies medicinais pode estar relacionado com as tarefas domésticas atribuídas a elas (BORGES et al., 2010), que geralmente são responsáveis pelo cuidado do lar e consequentemente a manutenção dos quintais, que foi o principal local citado para obtenção das plantas medicinais para consumo (MONTELES et al., 2007; OLIVEIRA et al., 2010; LIPORACCI et al., 2013).

Evidenciou-se que as pessoas mais idosas, com maior número de filhos e com baixa escolaridade conhecem e utilizam uma maior diversidade de plantas medicinais e que esse conhecimento é transmitido de forma oral, de uma geração para outra, estando de acordo com outros estudos (OLIVEIRA et al., 2012; MESSIAS et al., 2014; GONÇALVES et al.; SILVA et al., 2018). Enfatiza-se, que pessoas mais velhas tendem a conhecer uma maior diversidade de espécies medicinais úteis, em virtude do saber acumulado ao longo de suas vidas (FLOR et al., 2015).

Outro aspecto observado foi a correlação positiva entre o número de filhos e a quantidade de plantas citadas, ou seja, quanto mais filhos uma família tem, maior é o conhecimento da mesma em relação as plantas medicinais. Ainda nesse escopo, quando analisado o grau de escolaridade, percebe-se um paradoxo, quanto maior o grau de estudo, menor o conhecimento.

Embora os resultados indiquem que o conhecimento tradicional sobre o uso de plantas medicinais esteja sendo repassado para a geração seguinte, verificou-se que os jovens conhecem um menor número de plantas medicinais. Algumas hipóteses que explicariam este resultado: 1) pela falta de interesse dos jovens

${ }^{6}$ http://www.ibama.gov.br/flora/extinção.htm 
2) pela modernização dos meios de comunicação e 3) os idosos não estão repassando o conhecimento de forma correta, (MEDEIROS et al., 2004; TEKLEHAYMANOT, 2009; ZENEBE et al., 2012). Ressalta-se que a falta de interesse dos jovens pelas etnoespécies representa uma ameaça ao repasse de informações, podendo levar a perda e/ou desaparecimento do conhecimento sobre o uso popular das mesmas (CAJAIBA et al., 2016a).

Em corroboração com as pesquisas de Mosca et al. (2009), Zucchi et al. (2013) e Carvalho et al. (2015) o conhecimento sobre o uso caseiro de plantas medicinais, ocorre principalmente por meio de familiares. Em geral, os informantes relataram ter adquirido o conhecimento com a mãe, avós e/ou um parente mais velho, portanto, esses conhecimentos são adquiridos originalmente na própria região, e o fato de a maioria declararem-se oriundos do próprio estado do Maranhão, torna esta constatação ainda mais evidente, demostrando uma rica herança cultural sobre o uso tradicional das plantas medicinais nessa localidade (GIRALDI et al., 2010). Levando em consideração a perda eminente dos conhecimentos tradicionais relacionados às etnoespécies, este estudo se torna um documento útil, contribuindo para preservação e manutenção dos mesmos.

\section{CONCLUSÕES}

Esta pesquisa permitiu registrar o conhecimento tradicional e empírico dos moradores da região urbana do município de Buriticupu/MA, acerca do uso das plantas medicinais. Verificou-se que os entrevistados, de modo geral, afirmam utilizar as espécies medicinais como recurso primário para a cura de enfermidades mais comuns. Observou-se, também que a principal forma de transmissão de conhecimento é a verbal. No entanto, há uma preocupação quanto a preservação destes saberes pelas gerações futuras, pois se evidenciou que as pessoas mais jovens conhecem um número inferior de plantas medicinais. Deste modo, faz-se necessário a ampliação de estudos etnobotânico, para que esses saberes possam resgatados e ao mesmo tempo ser divulgada a importância preservação do mesmo para as gerações futuras.

\section{REFERÊNCIAS}

BARBOSA, C. K. R.; COSTA, J. P. R.; BOMFIN, F. P. G.; ALMEIDA, A. C.; MARTINS, E. R.. Qualidade microbiológica de plantas medicinais cultivadas e comercializadas em Montes Claros, MG. Biotemas, v.23, n.1, p.77-81, 2010. DOI: https://doi.org/10.5007/2175-7925.2010v23n1p77

BENNETT, B. C.; PRANCE, G. T.. Introduced plants in the indigenous pharmacopoeia of Northern South America. Economic Botany, v.54, n.1., p.90-102, 2000. DOI: https://doi.org/10.1007/BF02866603

BORGES, K. N.; BAUTISTA, H. P.. Etnobotânica de Plantas Medicinais na Comunidade de Cordoaria, Litoral Norte do Estado da Bahia. Plurais Revista Multidisciplinar, v.1, n.2, p.153-174, 2010.

BRASIL. Decreto n. $\mathbf{5 . 8 1 3}$ de 22 de junho de 2006. Dispõe sobre a Política Nacional de Plantas Medicinais e Fitoterápicos. Brasília: DOU, 2006.
BRITO, M. F. M.; LUCENA, R. F. P.; CRUZ, D. D. Conhecimento etnobotânico local sobre plantas medicinais: uma avaliação de índices quantitativos. Asociación Interciencia Caracas, v.40, n.3, p.156-164, 2015.

CAJAIBA, R. L.; SILVA, W. B.; SOUSA, R. D. N.; SOUSA, A. S.. Levantamento etnobotânico de plantas medicinais comercializadas no município de Uruará, Pará, Brasil. Biotemas, v.29, n.1, p.115-131, 2016a. DOI: https://doi.org/10.5007/2175-7925.2016v29n1p115

CAJAIBA, R. L.; GOMES, A. F.; MEDEIROS, R. R.; SILVA, W. B. Perfil dos comerciantes de plantas medicinais no município de Uruará, Pará, Brasil. Enciclopédia Biosfera, v.13, n.24, p.1473-1482, 2016b. DOI: https://doi.org/10.18677/EnciBio 2016B 136

CAJAIBA, R. L.; PEREIRA, K. S.; MARTINS, J. S. C.; SOUSA, E. S.; SILVA, W. B.. Megasoma actaeon (Linnaeus) (Scarabaeidae: Dynastinae): first record for Maranhão state, northeastern 
Brazil. Scientia Amazonia, v.8, p.13-16, 2019.

CARVALHO, A. P. S.; CONCEIÇÃO, G. M.. Utilização de plantas medicinais em uma área da estratégia de saúde da família, Caxias, Maranhão. Enciclopédia Biosfera, v.11, n.21, p.3478, 2015.

CARVALHO, M.C.G.; PIRES, R. L.; FLORINDO, W. S. CAVALCANTE, A. S. S.. Evidências para o uso de Indigo naturalis no tratamento da psoríase tipo placa: uma revisão sistemática. Natureza on line, v.8, n.3, p.127-131, 2010.

CAVALCANTE, J. W.; CAVALCANTE, V. M. G.; BIESKI, I. G. C. Conhecimento tradicional e etnofarmacológico da planta medicinal copaiba (Copaifera langsdorffii Desf.). Biodiversidade, v.16, n.2, p.123, 2017.

COSTA, R. A.. A identidade e o conhecimento etnobotânico dos moradores da Floresta Nacional do Amapá. Dissertação, Universidade Federal do Amapá, 2013.

FLOR, A. S. S. O.; BARBOSA, W. L. R.. Sabedoria popular no uso de plantas medicinais pelos moradores do bairro do sossego no distrito de Marudá, PA. Revista Brasileira de Plantas Medicinais, v.7, n.4, p.757-768, 2015. DOI: http://dx.doi.org/10.1590/1983-084X/14 064

FREITAS, A. V. L. F.; COELHO, M. F. B.; MAIA S. S. S.; AZEVEDO, A. B. R.. Plantas medicinais: um estudo etnobotânico nos quintais do Sítio Cruz, São Miguel, Rio Grande do Norte, Brasil. Brazilian Journal of Biosciences, v.10, n.1, p.48-59, 2012

GIL, A. C.. Método e técnicas de pesquisa social. 6 ed. São Paulo: Atlas, 2008.

GIRALDI, M.; HANAZAKI, N.. Uso e conhecimento tradicional de plantas medicinais no Sertão do Ribeirão, Florianópolis, SC, Brasil. Acta Botanica Brasilica, v.24, n.2, p.395-406, 2010. DOI: http://dx.doi.org/10.1590/S010233062010000200010.

GOLÇAVES, M. M. M.; CAJAIBA, R. L.; SANTOS, W. B.; SOUSA, E. S.; MARTINS, J. S. C.; PEREIRA, K. S.; SOUSA, V. A.. Estudo etnobotânico do conhecimento e uso de plantas medicinais em Santa Luzia, Maranhão, Brasil. Revista Ibero Americana de Ciências Ambientais, v.9, n.5, p.12-21, 2018. DOI: http://doi.org/10.6008/CBPC2179- 6858.2018.005.0002

GUARIN NETO, G.; MORAIS, R. G.. Recursos medicinais de espécies do cerrado de Mato Grosso: um estudo bibliográfico. Acta Botanica Brasilica, v.17, n.4, p.561-584, 2003. DOI: http://dx.doi.org/10.1590/S010233062003000400009

HAMMER, $\varnothing$.; HARPER, D. A. T.; RYAN, P. D.. Past: Paleontological Statistics Software Package for Education and Data Analysis. Palaeontologia Electronica, v.4, n.1, p.9, 2001.

IBGE. Instituto Brasileiro de Geografia e Estatística. População estimada: IBGE, Diretoria de Pesquisas, Coordenação de População e Indicadores Sociais, Estimativas da população residente com data de referência $1^{\circ}$ de julho de 2018. Rio de Janeiro: IBGE, 2018.

LEMOS, J. R.; ARAUJO, J. L.. Estudo etnobotânico sobre plantas medicinais na comunidade de Curral Velho, Luís Correia, Piauí, Brasil. Biotemas, v.28, n.2, p.125-136, 2015. DOI: https://doi.org/10.5007/2175-7925.2015v28n2p125

LIMA, J. S.; CAJAIBA, R. L.; MARTINS, J. S. C.; PEREIRA, K. S.; SOUSA, E. S.. Educação ambiental em resíduos sólidos em escolas no município de Buriticupu-MA. Scientia Amazonia, v.6, n.3, p.11-16, 2017.

LIPORACCI, H. S. N.; SIMÃO, D. G.. Levantamento etnobotânico de plantas medicinais nos quintais do Bairro Novo Horizonte, Ituiutaba, Revista Brasileira de Plantas Medicinais, Campinas, v15, n.4, p.529-540, 2013. DOI: http://dx.doi.org/10.1590/S1516-05722013000400009

MEDEIROS, M. F. T.; FONSECA, V. S.; ANDREATA, R. H. P. Plantas medicinais e seus usos pelos sitiantes da Reserva Rio das Pedras, Mangaratiba, RJ, Brasil. Acta Botanica Brasilica, v.18, n.2, p.391-399, 2004. DOI: http://dx.doi.org/10.1590/S0102-33062004000200019

MESSIAS, M. C. T. B.; MENEGATTO, M. F.; PRADO, A. C. C.; SANTOS, B. R.; GUIMARÃES, M. F. M.. Uso popular de plantas medicinais e perfil socioeconômico dos usuários: um estudo em área urbana em Ouro Preto, MG, Brasil. Revista Brasileira de Plantas Medicinais, v.17, n.1, p.76-104, 2015. DOI: http://dx.doi.org/10.1590/1983-084X/12 139

MONTELES, R.; PINHEIRO, C. U. B.. Plantas medicinais em um quilombo maranhense: uma perspectiva etnobotânica. Revista de Biologia e Ciências da Terra, v.7, n.2, 2007.

MOSCA, V. P.; LOIOLA, M. I. B.. Uso popular de plantas medicinais no Rio Grande do Norte, nordeste do Brasil. Revista Caatinga, v.22, n.4, p.225-234, 2009.

NETO, F. R. G.; ALMEIDA, G. S. S. A.; JESUS, N. G.; FONSECA, M. R.. Estudo Etnobotânico de plantas medicinais utilizadas pela Comunidade do Sisal no município de Catu, Bahia, Brasil. Revista Brasileira de Plantas Medicinais, v.16, n.4, p.856-865, 2014. DOI: http://dx.doi.org/10.1590/1983084X/11207

OLIVEIRA, A. P. C.. O conhecimento tradicional sobre plantas medicinais no âmbito da saúde da mulher: uma perspectiva no contexto do produto tradicional fitoterápico. Revista Fitos, v.10, n.4, p. 1-62, 2016. DOI: https://doi.org/10.5935/2446-4775.20160043

OLIVEIRA, E. R.; MENINI NETO, L.. Levantamento etnobotânico de plantas medicinais utilizadas pelos moradores do povoado de Manejo, Lima Duarte - MG. Revista Brasileira de Plantas Medicinais, v.14, n.1., p.31120, 2012. Dol: http://dx.doi.org/10.1590/S151605722012000200010

OLIVEIRA, G. L.; OLIVEIRA, A. F. M.; ANDRADE, L. H. C.. Plantas medicinais utilizadas na comunidade urbana de Muribeca, Nordeste do Brasil. Acta Botanica Brasilica, v.24, n.2, p.571-577, 2010. DOI: http://dx.doi.org/10.1590/S010233062010000200026

PINTO, E. P. P.; AMOROZO, M. C. M.; FURLAN, A. Conhecimento popular sobre plantas medicinais em comunidades rurais de mata atlântica - Itacaré, BA, Brasil. Acta Bototanica Brasilica, v.20, n.4, p.751-762, 2006. DOI: http://dx.doi.org/10.1590/S0102-33062006000400001 
POSSAMAI, R. M.. Levantamento etnobotânico das plantas de uso medicinal em Mariana Pimentel, RS. [Dissertação]. 131, 2018.

POSEY, D. A.. Entomologia de tribos indígenas da Amazônia. In: RIBEIRO, D.. Suma etnológica brasileira. Rio de Janeiro: Petrópolis Vozes/Finep, 1986. p.251-272.

ROCHA, A. R.; BOSCOLO, O. H.; FERNANDES, L. R. R. M. Etnobotânica: um instrumento para valorização e identificação de potenciais de proteção do conhecimento tradicional. Interações, v.16, n.1, p.67-74, 2015.

ROKAYA, M. B.; MÜNZBERGOVÁ, Z.; TIMSINA, B.. Ethnobotanical study of medicinal plants from the Humla district of western Nepal. Journal of Ethnopharmacology, v.130, n.3, p.485-504, 2010.

ROQUE, A. A.; ROCHA, R. M.; LOIOLA, M. I. B.. Uso e diversidade de plantas medicinais da Caatinga na comunidade rural de Laginhas, município de Caicó, Rio Grande do Norte (nordeste do Brasil). Revista Brasileira de Plantas Mededicinais, v.12, n.1, p.31-42, 2010. DOI: http://dx.doi.org/10.1590/S1516-05722010000100006

SANTOS, A. B. N.; ARAÚJO, M. P.; SOUSA, R. S.; LEMOS, J. R.. Plantas medicinais conhecidas na zona urbana de Cajueiro da Praia, Piauí, Nordeste do Brasil. Revista Brasileira de Plantas Medicinais, v.18, n.2, p.442-450, 2016. DOI: http://dx.doi.org/10.1590/1983-084X/15 149

SILVA, C. T. A.; MORAES F. L.; DARIVA, F. R.. Plantas Medicinais Utilizadas pela População do Município de Guaraniaçu - Paraná. Cultivando o Saber, v.4, n.2, p.53-70, 2011.

SILVA, W. B.; CAJAIBA, R. L.; PARRY, M. M.. Levantamento etnobotânico de plantas medicinais utilizadas pelos moradores do município de Uruará, estado do Pará, Brasil. Revista Cubana de Plantas Medicinales, v.22, n.4, 2017.

SEGAWA, P.; KASENENE, J. M.. Medicinal plant diversity and uses in the Sango bay area, Southern Uganda. Journal of Ethnopharmacology, v.113, n.3, p.521-540, 2007.
STEFANELLO, S.; KOZERA C.; RUPPELT, B. M.; FUMAGALLI, D.; CAMARGO, M. P.; SPONCIADO D.. Levantamento do uso de Plantas Medicinais na universidade federal do paraná, Palotina - PR, Brasil. Revista Extensão em Foco, n.15, p.15 27, 2018

TEKLEHAYMANOT, T.. Ethnobotanical study of knowledge and medicinal plants use by the people in Dek Island in Ethiopia. Journal of Ethnopharmacology, v.124, n.1, p.6978, 2009.

VÁSQUEZ, S. P. F.; MENDONÇA, M. S.; NODA, S. N.. Etnobotânica de plantas medicinais em comunidades ribeirinhas do município de Manacapuru, Amazonas, Brasil. Revista Acta Amazônica. v.44, n.4, p.457-472, 2014. DOI: http://dx.doi.org/10.1590/1809-4392201400423

VIEIRA, L. S.; SOUSA, R. S.; LEMOS, J. R.. Plantas medicinais conhecidas por especialistas locais de uma comunidade rural maranhense. Revista Brasileira de Plantas Medicinais, v.17, n.4, p.1061-1068, 2015. DOI: http://dx.doi.org/10.1590/1983-084x/15 009

WANDERLEY, L. S. M.; SILVA, L. V. L. A.; CEZAR, L. M.; DIAS, F. O. C.; GALDINO, P. K. S.; ARAÚJO, I. M.. Uso de plantas medicinais por indivíduos da comunidade do Valentina-PB. Rev. Ciêc. Saúde Nova Esperança, v.13, n.2, p.99-105, 2015. DOI: https://doi.org/10.17695/revcsnevol13n2p99\%20$\% 20105$

ZENEBE, G.; ZERIHUN, M.; SOLOMON, Z.. An Ethnobotanical Study of Medicinal Plants in Asgede Tsimbila District, Northwestern Tigray, Northern Ethiopia. Ethnobotany Research \& Applications, v.10, n.1, p.305-320, 2012.

ZAGO, L. M. S.. Vinte e dois anos de pesquisa sobre plantas medicinais: uma análise cienciométrica. Tecnia, v.3, n.1, 2018.

ZUCCHI, M. R.; OLIVEIRA JÚNIOR, V. F.; GUSSONI, M. A.; SILVA, M. B.; SILVA, F. C.; MARQUES, N. E.. Levantamento etnobotânico de plantas medicinais na cidade de Ipameri. Revista Brasileira de Plantas Medicinais, Campinas, v.15, n.2, p.273-279, 2013. DOI: http://dx.doi.org/10.1590/S1516$\underline{05722013000200016}$

A CBPC - Companhia Brasileira de Produção Científica (CNPJ: 11.221.422/0001-03) detém os direitos materiais desta publicação. Os direitos referem-se à publicação do trabalho em qualquer parte do mundo, incluindo os direitos às renovações, expansões e disseminações da contribuição, bem como outros direitos subsidiários. Todos os trabalhos publicados eletronicamente poderão posteriormente ser publicados em coletâneas impressas sob coordenação da Sustenere Publishing, da Companhia Brasileira de Produção Científica e seus parceiros autorizados. Os (as) autores (as) preservam os direitos autorais, mas não têm permissão par coordenção da Sustenere Publishing, da Companhia Brasileira de Produçăo cientíca e seus parce 